\title{
utilisation du microscope électronique à balayage en vue de la compréhension des mécanismes du gonflement des sols argileux
}

\author{
Jean-Pierre TROALEN \\ Assistant \\ Institut National Polytechnique de Lorraine, E.N.S.G. \\ Laboratoire de Mécanique et Hydraulique des Sols et des Roches* \\ Mahmoud ABOUSHOOK \\ Ingénieur-chercheur \\ Institut National Polytechnique de Lorraine, E.N.S.G. \\ Laboratoire de Mécanique et Hydraulique des Sols et des Roches* \\ Jean-Paul TISOT \\ Maître-assistant \\ Institut National Polytechnique de Lorraine, E.N.S.G. \\ Laboratoire de Mécanique et Hydraulique des Sols et des Roches*
}

\section{INTRODUCTION}

Le travail que nous exposons ici s'inscrit dans le cadre plus vaste d'une étude des propriétés physiques et mécaniques de matériaux argileux gonflants de la région du Caire (Egypte) oũ ils posent de nombreux problèmes d'ordre géotechnique. Cette étude fait suite à un certain nombre de travaux antérieurs et les complète $(1,3,4,5)$.

Les différents problèmes soulevés par la mesure des contraintes de gonflement à volume constant et par leur interprétation sont plus particulièrement abordés.

Les cinq matériaux que nous avons étudiés sont tout d'abord caractérisés du point de vue de leurs proprié- tés minéralogiques, chimiques et physiques, puis des essais de gonflement à la cellule triaxiale sont réalisés afin de mesurer les contraintes axiale et radiale de gonflement.

Les résultats obtenus à la cellule triaxiale ne peuvent pas être en totalité expliqués par les seules analyses de l'identification. Afin d'essayer de mieux cerner les mécanismes et les comportements observés, les auteurs ont réalisé une analyse détaillée des textures initiales et finales à l'aide du microscope électronique à balayage. Les résultats ainsi obtenus permettent d'établir une meilleure corrélation entre les paramètres physiques et

\footnotetext{
* Institut National Polytechnique de Lorraine, rue du Doyen Marcel Roubault, B.P. 40, 54501 Vandœuvre-lès-Nancy.
} 
mécaniques mesurés et, ainsi, il est possible de mieux interpréter et décrire les différents mécanismes du gonflement.

\section{CARACTÉRISATION DES MATÉRIAUX}

Du point de vue géologique, le sol et le sous-sol du district urbain du Caire (Egypte) sont constitués par des formations sableuses et/ou graveleuses, d'âge Eocène à Quaternaire récent.

Les formations attribuées à l'Oligocène, au Miocène et au Pliocène sont celles où les problèmes géotechniques et les désordres observés sur les constructions sont les plus fréquents. En effet, l'observation montre que dans ces terrains sableux et/ou graveleux il s'intercale de nombreux bancs ou lentilles silto-argileux. Ceux-ci sont la cause majeure des désordres observés.

Les cinq matériaux étudiés sont caractéristiques de ces niveaux. A l'échelle macroscopique, ils présentent deux types principaux de structure. Ce sont soit des argilites massives (échantillons $A, C$ et E) soit des argilites litées ou shales (échantillons B et D).

Les paramètres minéralogiques sont reportés sur le tableau I. Les analyses chimiques et les propriétés qui en découlent figurent sur le tableau II. Les principales propriêtés physiques sont données sur le tableau III

Du point de vue de leur nature, la composition minéralogique des cinq matériaux étudiés fait apparaître un mélange de quartz, de montmorillonite et de kaolinite. Les pourcentages relatifs de ces trois constituants majeurs varient considérablement d'un matériau à un autre. De plus, il apparaît que le type même de la montmorillonite peut changer. Il en est de même en ce qui concerne la granulométrie. Ceci est particulièrement évident pour l'échantillon $\mathrm{A}$.

Les propriétés chimiques et physiques seront commentées avec les résultats et l'interprétation des essais de gonflement à la cellule triaxiale ainsi qu'après les analyses réalisées au microscope électronique à balayage.

\section{ESSAIS DE GONFLEMENT A LA CELLULE TRIAXIALE}

La méthode que nous avons utilisée est celle qui a été mise au point dans des travaux antérieurs (5). Nous n'en présenterons ici qu'un résumé partiel.

Le but de cette méthode est de mesurer les contraintes axiale et radiale de gonflement dans les sols intacts en fonction de la variation de la teneur en eau en cours d'essai. La réalisation d'un tel essai nécessite l'utilisation d'une cellule triaxiale classique mais équipée d'un capteur de force (mesure de la contrainte axiale) et d'un capteur de pression (mesure de la contrainte radiale). L'essai débute par l'application d'une pression isotrope et d'une contre pression de faibles valeurs. On mesure les contraintes axiale et radiale en fonction de la teneur en eau au cours de l'essai, jusqu'à l'obtention d'une stabilisation. On définit comme étant les contraintes de gonflement, les valeurs des contraintes radiales et axiale à la fin de l'essai. De plus, il est réalisé des cycles entrée/sortie d'eau dans l'échantillon en cours d'essai. Ces cycles permettent l'observation d'éventuelles modifications des contraintes de gonflement.

Les résultats obtenus pour les cinq matériaux sont présentés sur le tableau IV et les figures 1 à 5 .

Ces résultats appellent un certain nombre de commentaires. Tout d'abord, il est évident que les valeurs des contraintes de gonflement obtenues sur les cinq matériaux ne sont pas semblables. D'autre part, le mécanisme «évolution des contraintes de gonflement » apparaît comme significativement différent d'un matériau à un autre, que ce soit le cas des argilites massives (échantillons A, C et E) ou celui des argilites litées (échantillons B et D).

Si nous tentons de relier ces observations aux paramèters physiques, chimiques et minéralogiques précédemment mesurés, il est possible de dire que pour les argilites massives ( $A, C, E)$, les contraintes de gonflement diminuent lorsque l'on effectue des cycles entrée/ sortie d'eau (fig. 2 et 3 ) sauf pour l'échantillon A (fig. 1) où la contrainte axiale augmente. La forme et la pente des cycles entrée/sortie d'eau n'évoluent pas selon le même mécanisme dans les trois matériaux. Les analyses minéralogiques et chimiques (tableaux I et II) font apparaître des différences dans la composition de ces matériaux. Les échantillons $A$ et $C$ possèdent $25 \%$ de minéraux argileux (classification dimensionnelle) alors que l'échantillon $\mathrm{E}$ en contient près de $40 \%$. De plus, l'échantillon a contient un plus fort pourcentage en montmorillonite dont la cristallinité est

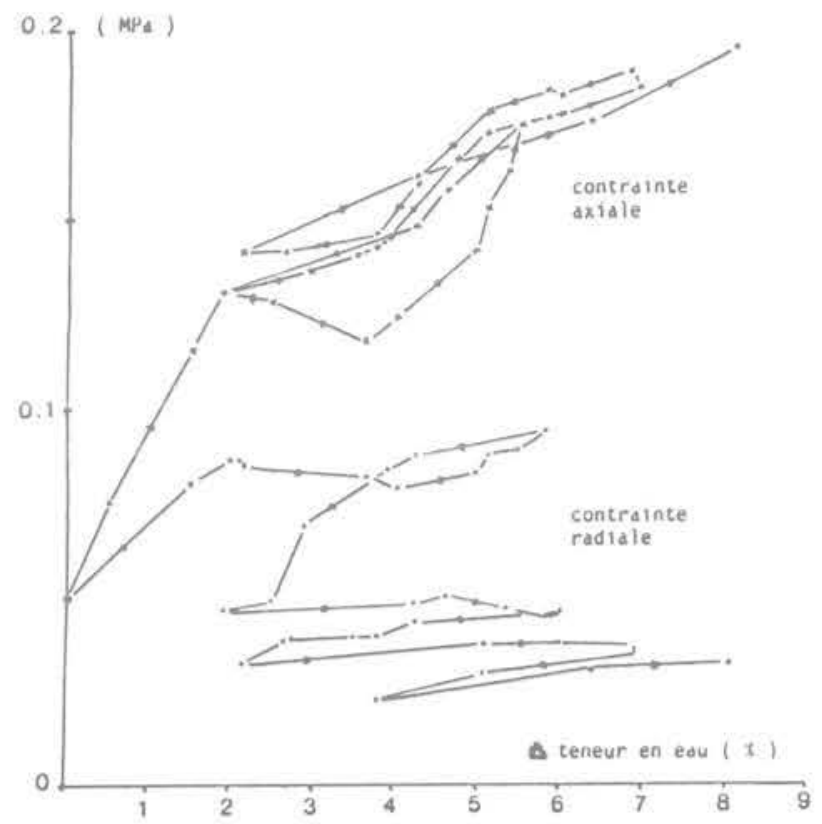

Fig. 1. - Echantillon A argilite massive. 


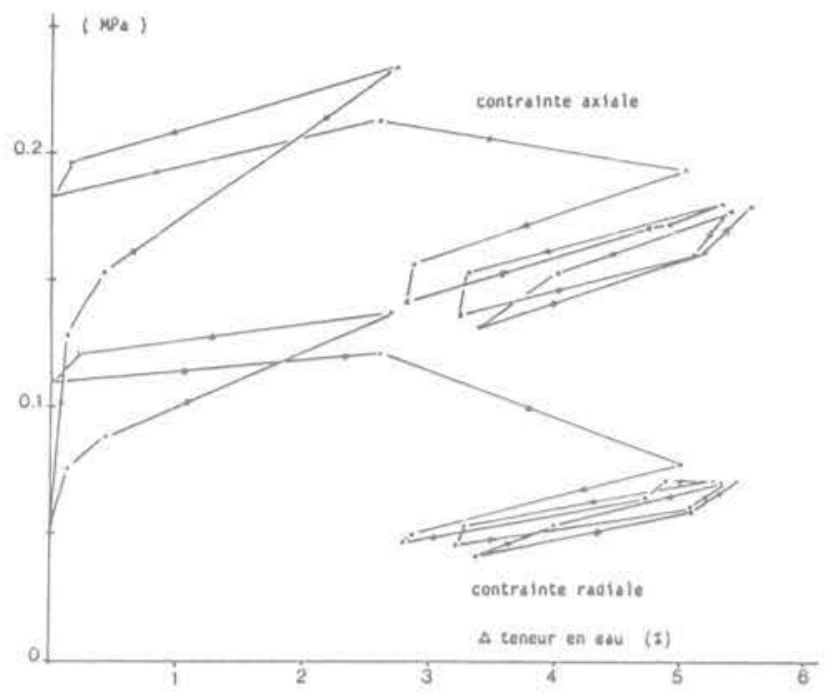

Fig. 2. - Echantillon $C$ argilite massive.

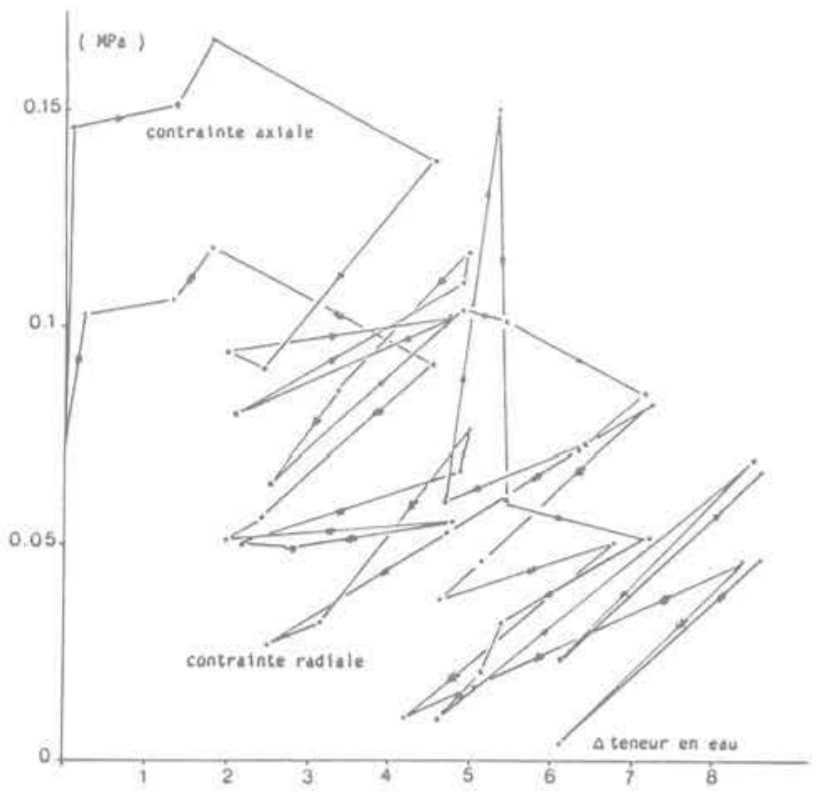

Fig. 3. - Echantillon E argilite massive.

plus élevée que celle des échantillons $\mathrm{C}$ et $\mathrm{E}$ (cristallinité supérieure à 2 microns, tableaux I et II). L'activité "bleu de méthylène " (activité 4 , tableau III) et l'activité « gonflement libre * (activité 3, tableau III) montrent que l'échantillon A présente des valeurs élevées et très différentes de celles obtenues pour les échantillons C et $E$.

Dans le cas des argilites litées ou shales (B, D), les valeurs des contraintes radiales de gonflement restent relativement constantes au cours de l'essai alors que les contraintes axiales de gonflement augmentent pendant les mêmes cycles entrée/sortie d'eau avant de se stabiliser. Cette stabilisation peut être précoce (fig. 5, échantillon D) ou plus tardive (fig. 4, échantillon B). Les analyses chimiques et minéralogiques (tableaux I et II) ne montrent pas de différence significative dans les

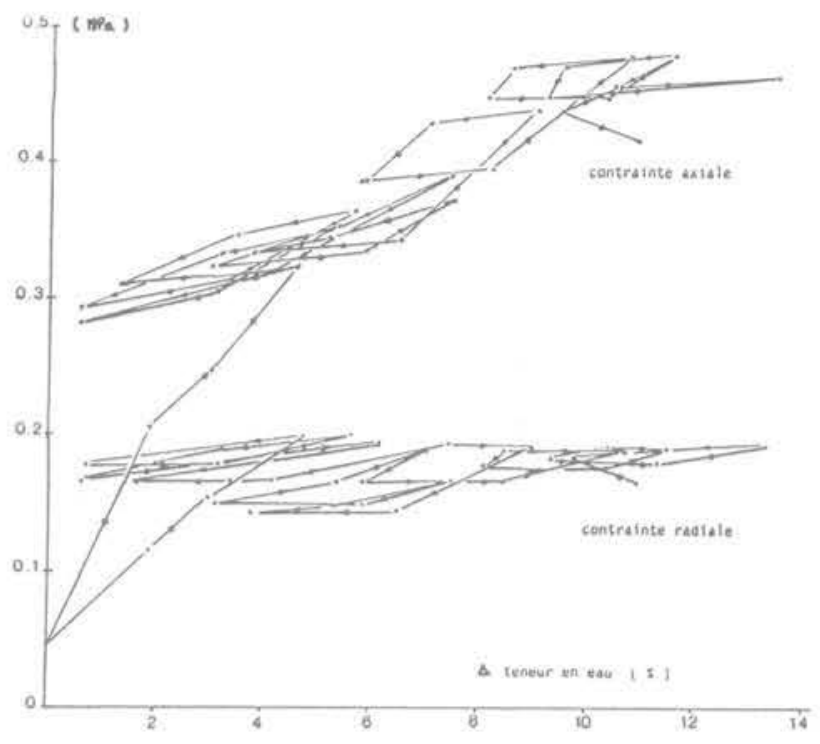

Fig. 4. - Echantillon B argilite litée.

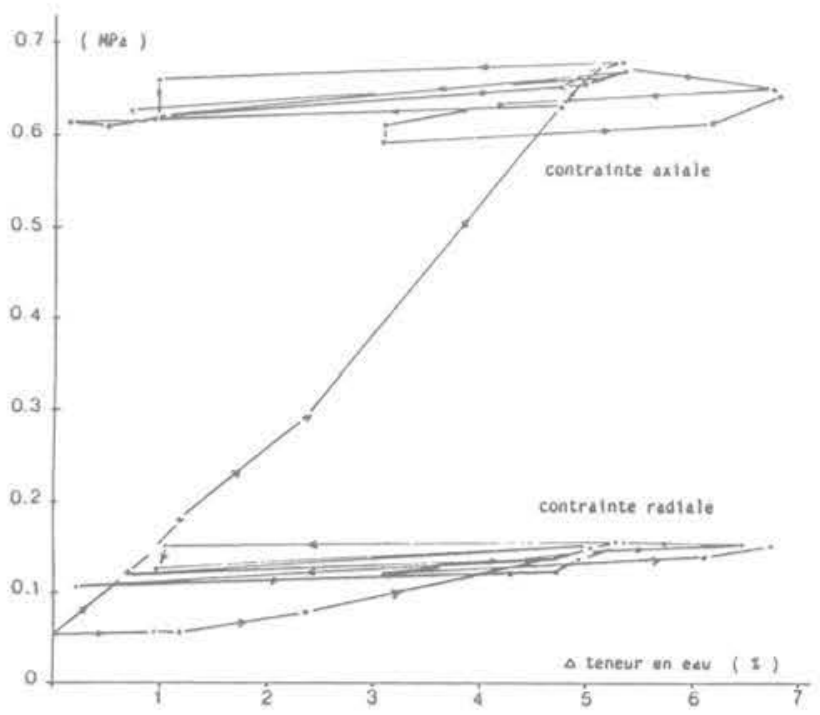

Fig. 5. - Echantillon $D$ argilite litée.

compositions. Il en est de même en ce qui concerne les propriétés physiques (tableau III), par exemple, les valeurs des activités restent semblables.

A ce stade de l'analyse, il est important de rappeler que les essais de gonflement à la cellule triaxiale sont des essais à volume constant (5), c'est-à-dire que les échantillons conservent non seulement le même volume, mais encore la même forme tout au long des essais (éprouvettes cylindriques de $35 \mathrm{~mm}$ de diamètre et d'élancement 2), même en cours de cycle entréel sortie d'eau. Il n'en est pas moins observé des comportements de gonflement différents. Si les paramètres physiques, chimiques et minéralogiques ne permettent pas d'expliquer en totalité ces mécanismes différents, c'est donc au niveau des textures qu'il faut, raisonnablement, rechercher une explication de ce phénomène. 
Tableau I. - Caractéristiques géologiques et minéralogiques.

\begin{tabular}{|c|c|c|c|c|c|c|c|c|c|}
\hline \multirow{2}{*}{$\begin{array}{l}2 \\
\frac{2}{3} \\
\frac{3}{2} \\
\frac{5}{2}\end{array}$} & \multirow{2}{*}{ 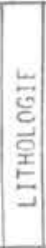 } & \multirow{2}{*}{ से } & \multirow{2}{*}{ 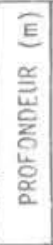 } & \multicolumn{2}{|c|}{ CARACTERISTIQUES GEOLOGIQUES } & \multicolumn{4}{|c|}{ CARACTERISTIQUES MINERALOGIQUES } \\
\hline & & & & Description & $\begin{array}{l}\text { Couleur } \\
\text { (Code Munse11) }\end{array}$ & $\begin{array}{l}\text { Minẻraux argileux } \\
\text { (\%) }\end{array}$ & $\begin{array}{r}\text { Minéraux non } \\
\qquad(\%)\end{array}$ & & ileux \\
\hline E & $\begin{array}{c}3 \\
\because \\
\because \\
\cdots\end{array}$ & 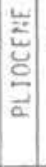 & $\frac{m}{1}$ & $\begin{array}{l}\text { ARGILITE MASSIVE : mélange } \\
\text { hétēronêne de sablons, sillts } \\
\text { et argiles; présence de } \mathrm{CaCO}_{3} \text {. }\end{array}$ & $\begin{aligned} \text { état } & \text { sec : } \\
7.5 \text { YR } & 3.5 / 4 \\
\text { êtat humide } & : \\
7.5 \text { YR } & 5.5 / 4\end{aligned}$ & 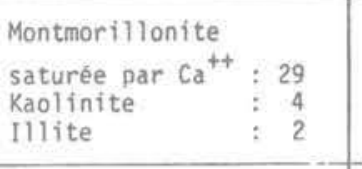 & $\begin{array}{l}\text { Quartz } \\
\text { Calcite } \\
\text { Oxydes de } \mathrm{Fe} \\
\text { Oxydes de } \mathrm{Mn} \\
\text { Halite }\end{array}$ & $\begin{array}{l}\vdots \\
\vdots \\
\vdots\end{array}$ & $\begin{array}{r}40 \\
16 \\
6 \\
1 \\
3\end{array}$ \\
\hline 3 & - & & $\stackrel{c}{i}$ & $\begin{array}{l}\text { ARGILITE LITEE : mélange } \\
\text { homogène de silts et d' } \\
\text { arqiles; présence de gypse. }\end{array}$ & $\begin{array}{l}\text { état } \sec : \\
5 \text { Y } 5 / 1 \\
\text { état humide : } \\
10 \text { YR } 5.5 / 3\end{array}$ & $\begin{array}{l}\text { Montmorillonite } \\
\text { saturée par } \mathrm{Na}^{+}: 40 \\
\text { Kaolinite }\end{array}$ & $\begin{array}{l}\text { Quartz } \\
\text { Feldspaths } \\
\text { Oxydes de } \mathrm{Fe} \\
\text { Oxydes de } \mathrm{Mn} \\
\text { Gypse } \\
\text { Halite }\end{array}$ & $\begin{array}{l}\vdots \\
\vdots \\
\vdots\end{array}$ & $\begin{array}{r}24 \\
10 \\
5 \\
3 \\
3 \\
2 \\
\end{array}$ \\
\hline$c$ & $\left(\begin{array}{c}\cdots \\
\because \\
\vdots \\
\vdots \\
\hdashline \\
\hdashline\end{array}\right.$ & & $\dot{m}$ & $\begin{array}{l}\text { ARGILITE MASSIVE : mélange } \\
\text { hétérogène de sablons, siltts } \\
\text { et argiles. }\end{array}$ & $\begin{aligned} \text { état } & \text { sec : } \\
& 5 \mathrm{GY} 5 / 1 \text { et } \\
& 2.5 \text { Y } 5 / 1 \\
& \text { état humide : } \\
& 2.5 \text { Y } 6 / 4\end{aligned}$ & $\begin{array}{l}\text { Montmorillonite }{ }^{21} \\
\text { saturée par } \mathrm{Na}^{+}, \mathrm{Ca}^{++} \\
\text {Kaolinite }\end{array}$ & $\begin{array}{l}\text { Quartz } \\
\text { Feldspaths } \\
\text { Oxydes de Fe } \\
\text { Oxydes de Mn }\end{array}$ & $\begin{array}{l}\vdots \\
\vdots \\
\vdots\end{array}$ & $\begin{array}{r}53 \\
10 \\
5 \\
7\end{array}$ \\
\hline $\mathrm{R}$ & 2 & 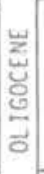 & $\begin{array}{c}m \\
1 \\
-1\end{array}$ & $\begin{array}{l}\text { ARGILITE LITEE : mélange non } \\
\text { homogène de silts et d'argiles } \\
\text { présence de gypse. }\end{array}$ & $\begin{array}{l}\text { état } \sec : \\
2.5 \text { y } 6 / 4 \text { et } \\
5 \text { YR } 5 / 8 \\
\text { état humide : } \\
10 \text { YR } 1 / 2\end{array}$ & $\begin{array}{l}\text { Montmorillonite } \quad 34 \\
\text { saturée par } \mathrm{Na}^{+}, \mathrm{Ca}^{++} \\
\text {Kaolinite } \quad: 21\end{array}$ & $\begin{array}{l}\text { Quartz } \\
\text { Oxydes de } \mathrm{Fe} \\
\text { Oxydes de } \mathrm{Mn} \\
\text { Gypse }\end{array}$ & $\begin{array}{l}\vdots \\
\vdots \\
\vdots\end{array}$ & $\begin{array}{r}35 \\
3 \\
4 \\
3\end{array}$ \\
\hline A & $\begin{array}{c}\ddots \\
\vdots \\
\vdots \\
\vdots \\
\vdots\end{array}$ & & $\begin{array}{l}\infty \\
1 \\
\infty\end{array}$ & $\begin{array}{l}\text { ARGILITE MASSIVE : mélange } \\
\text { hétérogène de sablons, silts } \\
\text { et argiles. }\end{array}$ & $\begin{array}{l}\text { etat } \sec : \\
5,10 \text { YR } 4,5 / 6 \\
\text { état humide: } \\
10 \text { YR } 4,5 / 3\end{array}$ & $\begin{array}{l}\text { Montmorillonite saturée } \\
\text { par } \mathrm{Na}^{+}, \mathrm{Ca}^{++} \\
25 \text { inf. à } 2 \text { microns } \\
50 \text { sup. a } 2 \text { microns }\end{array}$ & $\begin{array}{l}\text { Quartz } \\
\text { Feldspaths } \\
\text { Oxydes de Fe } \\
\text { Oxydes de } \mathrm{Mn}\end{array}$ & $\begin{array}{l}\vdots \\
\vdots\end{array}$ & $\begin{array}{r}7 \\
10 \\
3 \\
5\end{array}$ \\
\hline
\end{tabular}

En effet, les analyses chimiques, minéralogiques et la mesure des propriétés physiques n'incluent pas le paramètre de texture. Ainsi, une composition identique ne signifie pas pour autant une identité dans la répartition spatiale des particules constituantes et, de ce fait le comportement au gonflement ou tout comportement mécanique n'est pas obligatoirement semblable et réciproquement $(7,8)$. C'est pourquoi, nous avons effectué des observations au microscope électronique à balayage sur les cinq matériaux étudiés. Les textures initiales (avant gonflement) et finales (parés gonflement) ont été observées dans les mêmes conditions de préparation.

\section{ANALYSE AU MICROSCOPE ÉLECTRONIQUE A BALAYAGE}

Les cinq matériaux sur lesquels ont été effectués les essais de gonflement ont été observés au microscope électronique à balayage et comparés aux échantillons témoins.

Compte tenu des hétérogénéités d'ensemble de ces matériaux et des surfaces petites et partielles que l'on observe au microscope électronique à balayage, il convient de prendre certaines restrictions dans l'exploitation des résultats.
A l'état initial (avant essais de gonflement), plusieurs types de microtextures paraissent se dégager, aussi bien dans les argilites massives (sols $A, C$ et E) que dans les argilites litées (sols B et D).

Pour l'échantillon A, la microtexture qui apparaît comme la plus plausible est constituée par des «agrégats» argileux de dimensions variées et séparés fréquemment par des discontinuités dont l'ouverture peut atteindre 2 à 2,5 microns. Celles-ci relient entre elles des «microcavités» de diamètre proche de 10 microns et dont la répartition est aléatoire (photographie 1 et 2). Dans les «agrégats» argileux il faut individualiser deux types qui s'imbriquent entre eux de façon également aléatoire. Le premier type peut être attribué à un assemblage de montmorillonite en feuillets empilés (photographie 3). L'espacement entre les feuillets est d'environ 0,3 micron et la largeur apparente du feuillet est voisine de 5 microns. Le second type est également un assemblage de feuillets de montmorillonite dont les empilements foliés s'organisent en microstructure type "nids d'abeille» (photographie 4). Les dimensions des feuillets semblent plus réduites que dans le premier type (entre 0,5 et 1 micron) et, l'ouverture du nid est de même ordre de grandeur.

Pour l'échantillon C, la microtexture apparaît comme une matrice argileuse relativement homogène, mais lâche. Les assemblages de feuillets de kaolinite/montmorillonite semblent intimement liés. Une dimension 
Tableau II. - Caractéristiques chimiques.

\begin{tabular}{|c|c|c|c|c|c|}
\hline & & $\mathrm{ECH}$ & TILLONS & & \\
\hline & A & B & $c$ & D & $E$ \\
\hline $\mathrm{SiO}_{2}$ & 49.0 & 59.0 & 77.0 & 57.0 & 67.0 \\
\hline $\mathrm{Al}_{2} \mathrm{O}_{3}$ & 14.0 & 18.5 & 8.5 & 16.0 & 8.2 \\
\hline $\mathrm{Fe}_{2} \mathrm{O}_{3}$ & 12.1 & 5.7 & 4.1 & 7.7 & 4.8 \\
\hline $\mathrm{MnO}$ & 0.12 & 0.03 & 0.02 & 0.05 & 0.04 \\
\hline $\mathrm{MgO}$ & 3.1 & 0.86 & 0.83 & 1.65 & 1.8 \\
\hline $\mathrm{CaO}$ & 1.5 & 0.65 & 0.55 & 0.85 & 5.2 \\
\hline $\mathrm{Na}_{2} \mathrm{O}$ & 2.45 & 0.68 & 0.70 & 2.35 & 0.55 \\
\hline $\mathrm{K}_{2} \mathrm{O}$ & 0.71 & 0.75 & 1.1 & 1.13 & 0.87 \\
\hline $\mathrm{THO}_{2}$ & 2,6 & 1.2 & 0.9 & 1.5 & 0.9 \\
\hline p.f. & 10.8 & 7.2 & 4.9 & 7.22 & 5.7 \\
\hline total & 96.4 & 94.7 & 98.6 & 95.5 & 95.1 \\
\hline
\end{tabular}

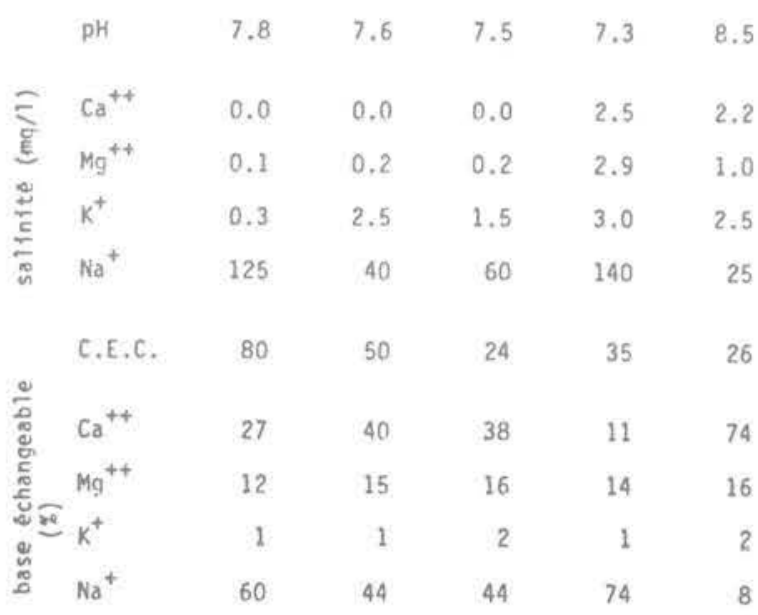

$\begin{array}{llllll}\text { E.S.P. } & 68.8 & 50 & 52.1 & 151.4 & 16.2\end{array}$
Tableau III. - Caractéristiques physiques.

ECHANTILLONS

POIOS VOLUMIOUES

$\left(\mathrm{kN} / \mathrm{m}^{3}\right)$

TENEUR EN EAU $(q)$

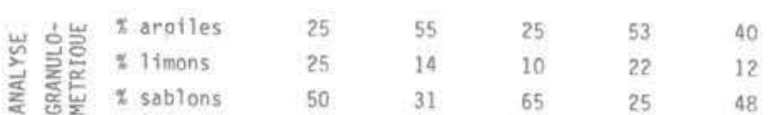

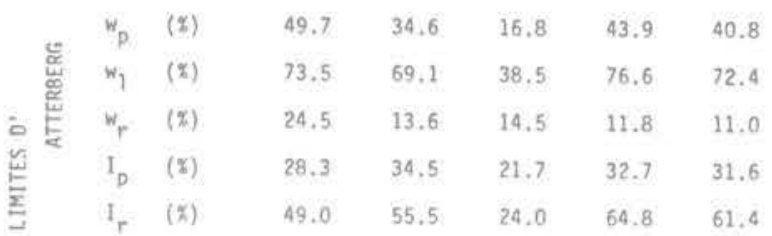

$\begin{array}{llllll}\text { GONFLEMENT LIBRE }(*) & 177.5 & 107.5 & 115.0 & 138.8 & 110.0\end{array}$

$\begin{array}{llllll}\text { VALEUR AU BLEU }\left(\mathrm{cm}^{3} / 0\right) & 12.1 & 13.0 & 8.0 & 13.6 & 10.1\end{array}$

$\begin{array}{llllll}\text { ACTIVITE } 1 & 0.95 & 0.63 & 0.87 & 0.62 & 0.79\end{array}$

$\begin{array}{llllll}\text { ACTIVITE ? } & 1.95 & 1.01 & 0.96 & 1.22 & 1.54\end{array}$

$\begin{array}{llllll}\text { ACTIVITE } 3 & 67.5 & 20.7 & 41.4 & 28.2 & 25.7\end{array}$

$\begin{array}{llllll}\text { ACTIVITE } 4 & 48.2 & 23.3 & 32.0 & 25.6 & 25.3\end{array}$

Activité 1 (SKEMPTON, 1953): activité 2 (RANGANATHAM \& SATYANARAYANA

1965); activite 3, définie par les auteurs: \% du gonflement libre défini par HOLTZ \& GIBBS (1956) / \% argiles inf. à 2 microns; activité 4 (IRAN-NGNO-CLAN, 1977)

Capacité d'échange C.E.C. (meq/100q); Pourcentage de

Na échangé (Na/C.E.C) $=$ E.S.P., d'aprés MITCHELL (1973)

Tableau IV. - Contraintes de gonflement et cycles entrée/sortie d'eau.

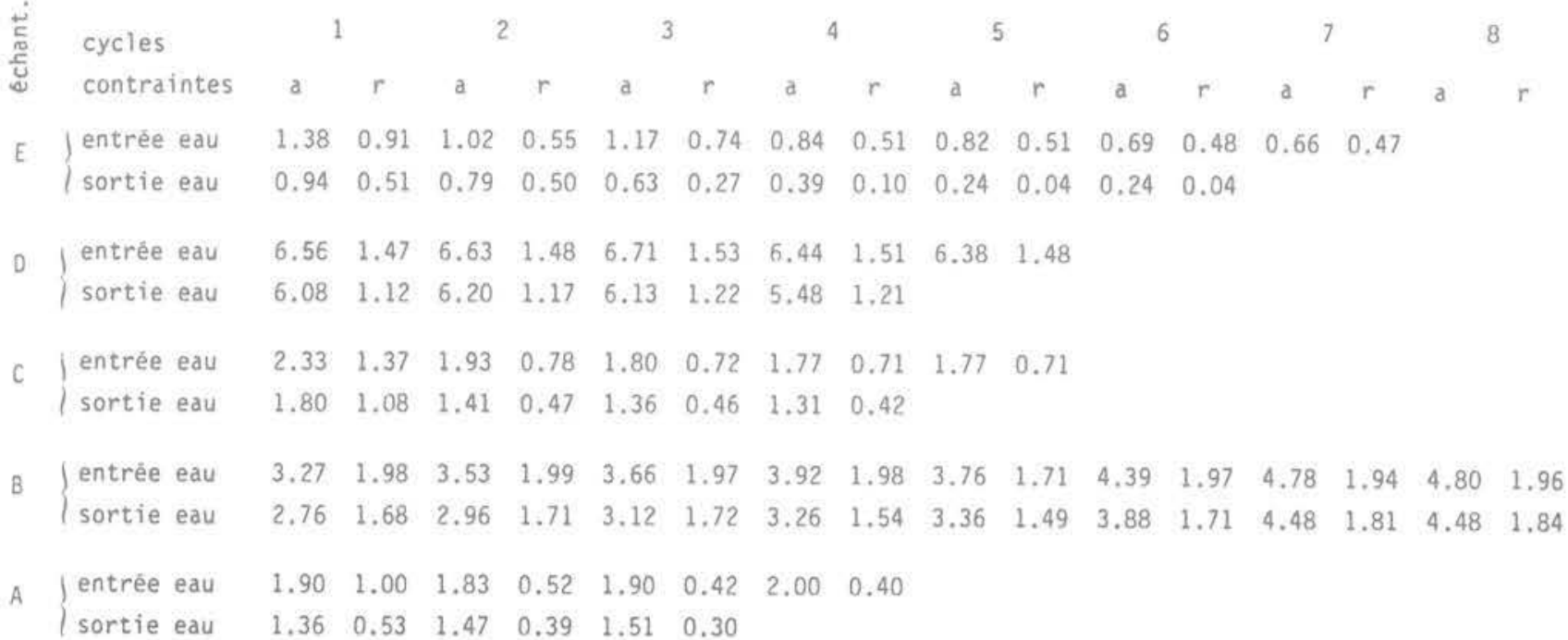

contraintes de gonflement à la cellule triaxiale: a = contrainte axiale; $r=$ contrainte radiale. 

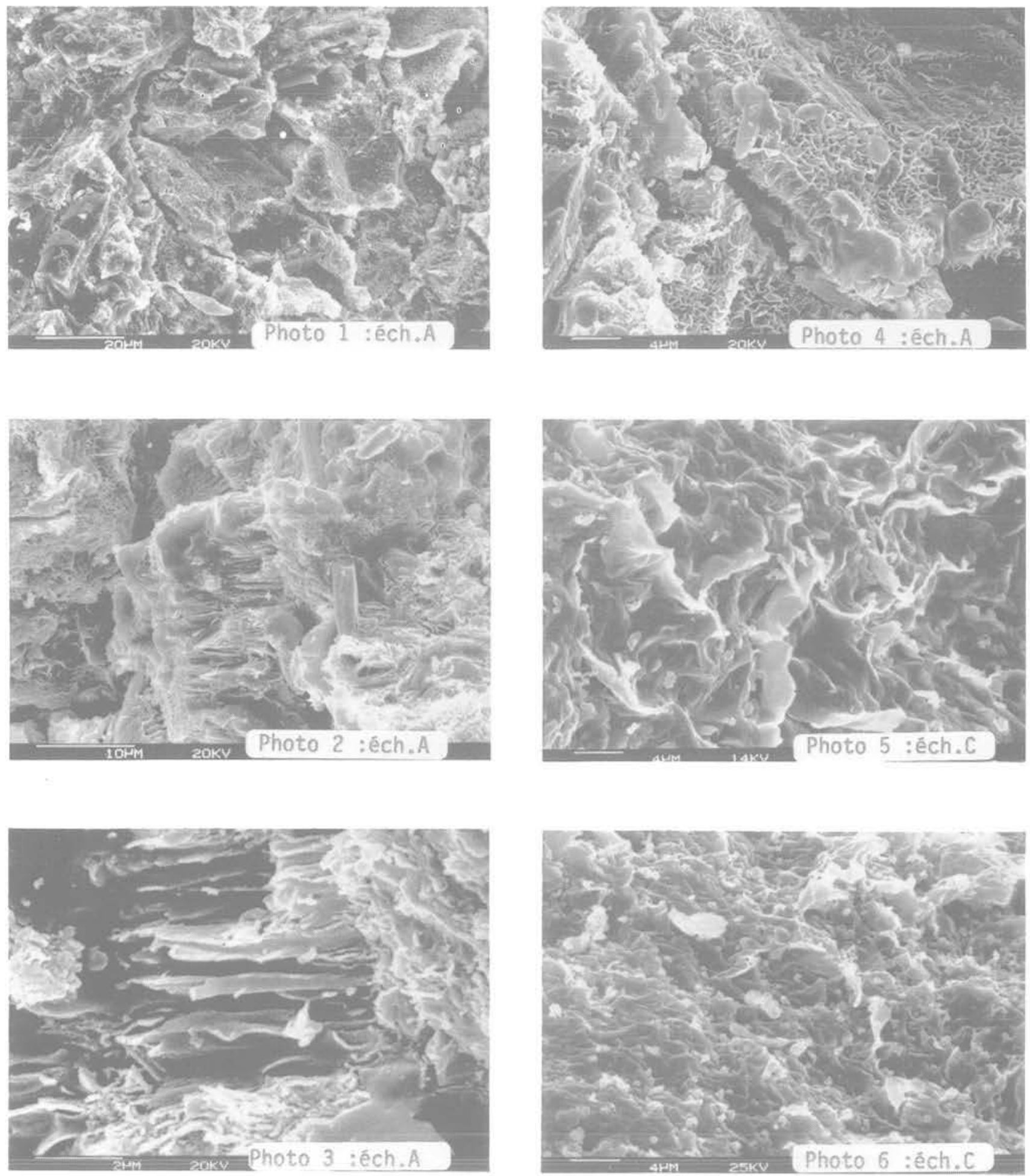

Microscope électronique à balayage:

moyenne des feuillets, sans distinction minéralogique, peut cependant être donnée: 6 à 8 microns (photographie 5). Il apparaît souvent des silts (essentiellement des quartz) au sein de cette microtexture. Leur taille est d'environ 3 à 4 microns, mais le plus souvent nous n'en observons que l'empreinte dans la matrice argileuse (7).

Dans le cas de l'échantillon $\mathrm{E}$, il existe une microtexture assez irrégulière, mais d'aspect dense. Les «agrégats " de kaolinite, illite et montmorillonite, de dimensions variées, s'articulent plus ou moins bien entre eux créant des «microcavités " plus ou moins isodiamétrique avec une ouverture comprise entre 5 et 10 microns (photographies 7 et 8). Il n'apparâ̂t pas 

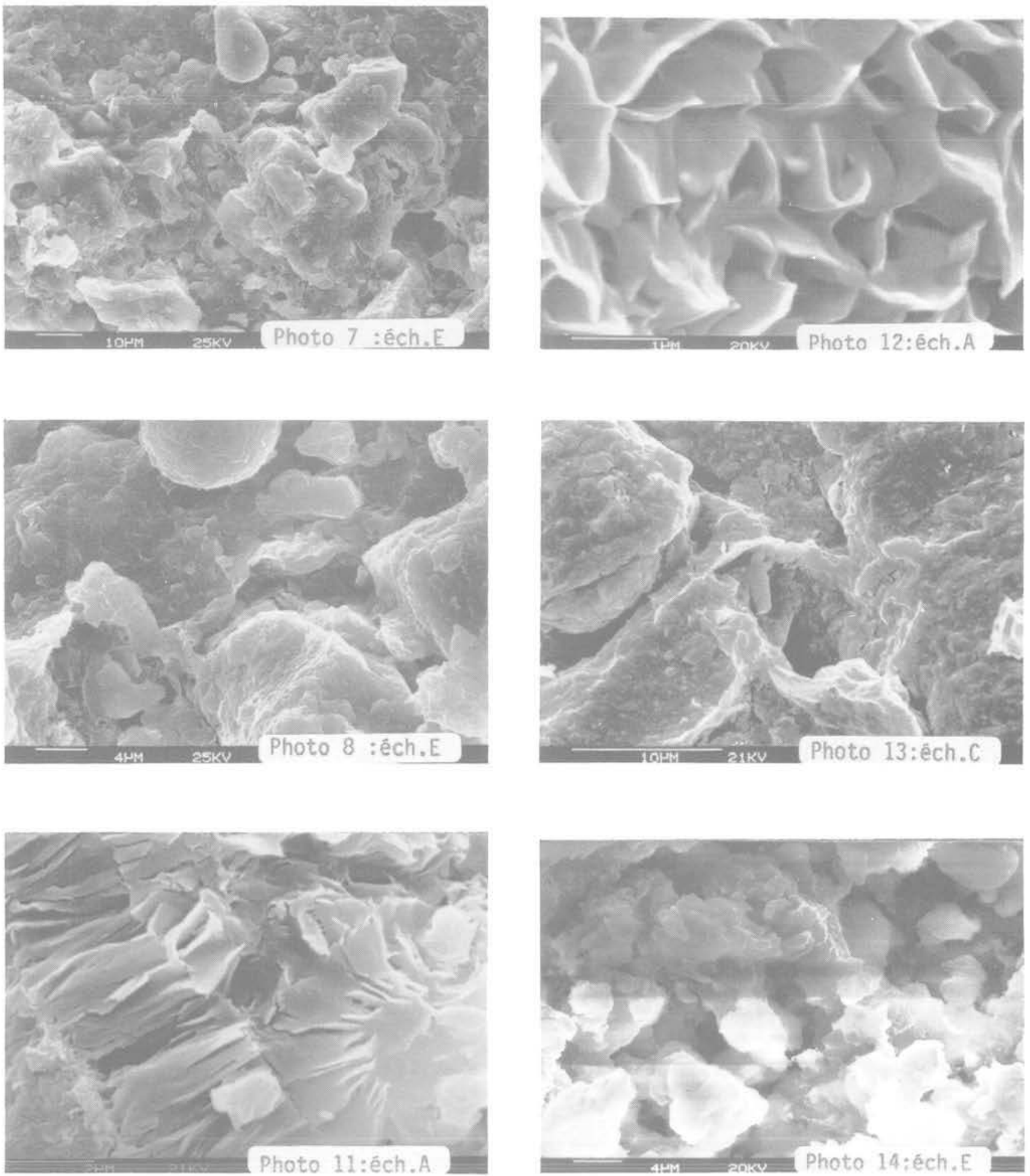

argilites massives, texture initiale.

d'ouverture entre les feuillets constitutifs des agrégats argileux observés.

Pour les argilites litées (sols B et D), nous observons également sur l'échantillon D une microtexture par des "agrégats * de kaolinite/montmorillonite séparés par d'assez nombreuses discontinuités (photographie 9).
L'ouverture des discontinuités peut atteindre 1 à 1,5 micron et, dans les zones où elles se raccordent (microcavités) l'ouverture peut atteindre 5 microns.

Pour l'échantillon B, la microtexture apparaît plus régulière que dans le cas du sol $\mathrm{D}$; en effet, les discontinuités y sont peu fréquentes et la dimension 

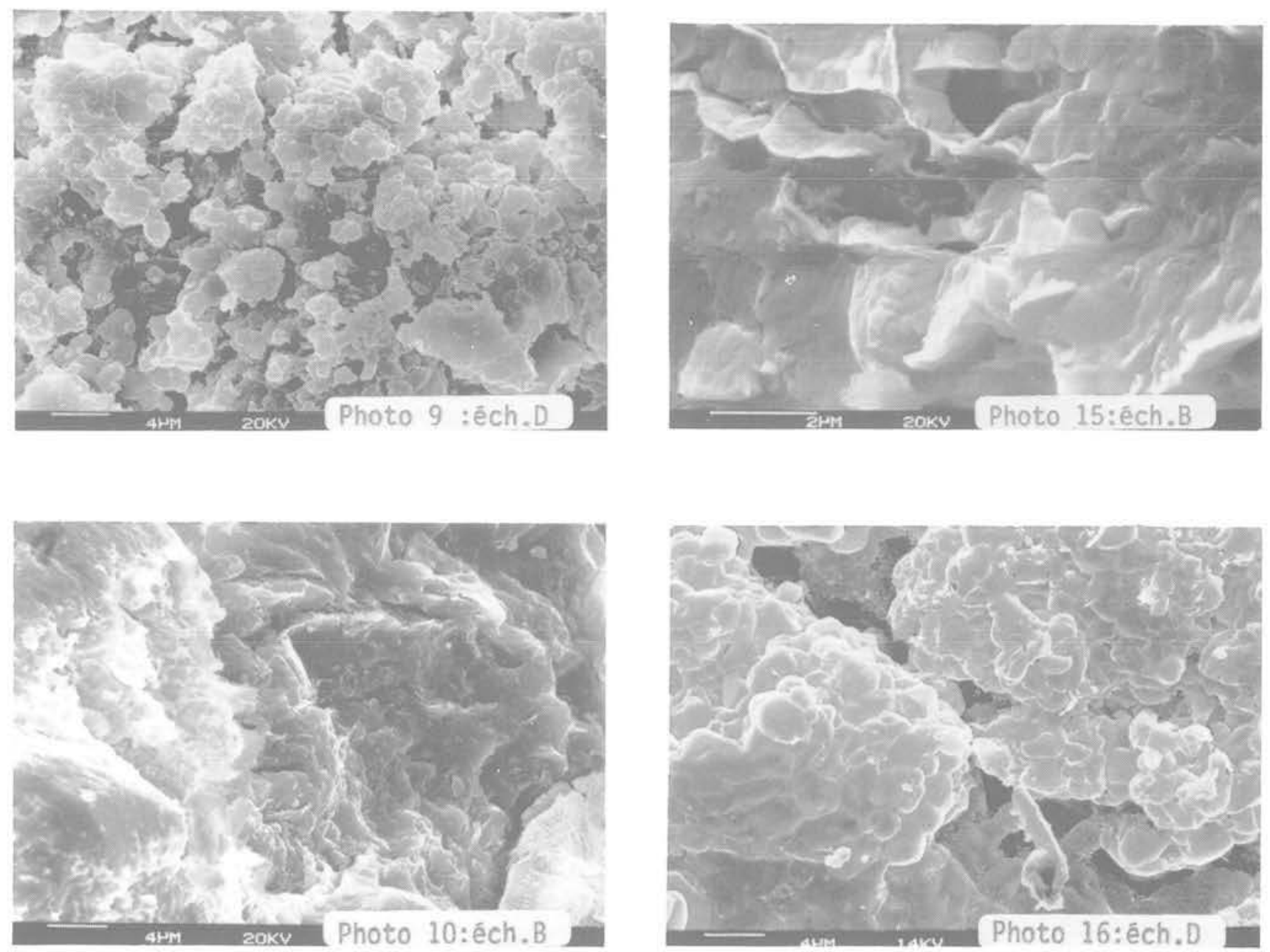

Microscope électronique à balayage: argilites litées, texture initiale.

moyenne des agrégats kaolinite/montmorillonite est environ 20 microns (photographie 10).

A l'état final (après essais de gonflement), les restrictions précédentes quant à la représentativité des échantillons au microscope électronique à balayage sont à rappeler, d'autant plus que les surfaces après gonflement et les surfaces témoins ne sont pas analysées et observées sur les mêmes plots pour des raisons évidentes de réalisation des dites surfaces d'observation. Nous pouvons cependant dégager un certain nombre d'observations.

Dans le cas de l'échantillon A, l'ouverture des feuillets de montmorillonite empilés (photographie 2 et 3 ) semble augmenter et pouvoir atteindre 0,6 microns (photographie 11). Dans la microtexture type «nids d'abeille» (photographie 4), on semble devoir là aussi assister à une certaine homogénéisation de l'assemblage argileux qui devient, de ce fait, plus régulier (photographie 12). Dans l'ensemble de l'échantillon A, l'augmentation de volume liée à l'expansion des feuillets se trouve compensée par une fermeture partielle ou totale des discontinuités préexistantes. Ceci est à mettre en rapport avec les conditions d'essai (gonflement à volume externe constant).

Dans le cas de l'échantillon $\mathrm{C}$, nous ne retrouvons plus, semble-t-il, la microtexture initiale. La matrice argileuse paraît encore plus dense malgré l'apparition d'un réseau de discontinuités (photographie 13). Les agrégats de kaolinite/montmorillonite s'articulent assez bien les uns aux autres. Le réseau de discontinuités semble être le résultat du réarrangement des agrégats initiaux (matrice argileuse plus lâche). Ce réarrangement est à mettre au compte de la stabilité du volume externe.

En ce qui concerne l'échantillon $E$ (photographie 14), la microtexture initiale paraît être conservée mais avec un réseau de discontinuités plus important. L'ouverture des discontinuités ne semble pas en revanche être plus grande, mais la dimension moyenne des agrégats argileux est en diminution (environ 10 microns). Cet échantillon correspond à un sol pour lequel les contraintes développées sont moins fortes. Il apparaît donc logique de constater des réajustements de microtexture moins importants que pour les échantillons des 
sols $\mathrm{A}$ et $\mathrm{C}$ dans lesquels les contraintes de gonflement sont plus fortes (notamment pour le sol A).

Pour les argilites litées (sols B et $\mathrm{C}$ ), l'aspect général traduit une modification des microtextures initiales. Pour le sol B (photographie 15), cela se manifeste par une ouverture entre les feuillets et montmorillonite (environ 1,5 microns) mais la dimension moyenne de l'agrégat paraît rester la même qu'à l'origine (soit environ 20 microns). L'ouverture entre les feuillets est compensée par la fermeture partielle ou totale des discontinuités initiales. Ceci est aussi à corréler avec les conditions expérimentales.

Dans le cas du sol D, l'aspect d'ensemble de la microtexture semble peu modifié à la limite près que le réseau de discontinuités tend à disparaître et que les agrégats augmentent en dimensions (photographie 16). Les microcavités sont elles aussi moins nombreuses, mais leur ouverture est généralement plus importante (souvent proche de 10 microns). Dans le cas du sol D, on obtient une stabilisation rapide des contraintes de gonflement au cours des cycles alors que pour le sol B il est nécessaire de réaliser un nombre important de cycles avant d'atteindre la stabilisation. La structure du sol $D$ est donc plus sensible aux phénomènes de gonflement que celle du sol B.

\section{CONCLUSION}

La connaissance des microtextures des matériaux argileux gonflants que nous avons étudiés, permet de mettre en évidence des mécanismes et des comportements différents que les paramètres physiques, chimiques, minéralogiques et mécaniques ne pouvaient à eux seuls expliquer. Ainsi, une identité de composition ou de paramètres physiques ne signifie pas une identité de comportement lors du gonflement et, réciproquement (7).

Dans le cas des argilites massives (A, C, E), les paramètres physiques, chimiques et minéralogiques montrent des différences entre ces trois matériaux. L'observation au microscope électronique à balayage en montrant des microtextures différentes confirme ces faits. Ainsi, après gonflement, le comportement de l'échantillon A peut être expliqué par la création d'une microtexture finale, serrée dans une direction et plus lâche dans une autre. De ce fait, c'est le réseau de discontinuité qui est essentiellement responsable du comportement observé en A. Dans le cas des échantillons $\mathrm{C}$ et $\mathrm{E}$, c'est le réarrangement des agrégats argileux (diminution de taille) et leur nouvelle distribution spatiale qui interviennent. Cette modification de la microtexture peut expliquer l'augmentation de la contrainte axiale et la diminution de la contrainte radiale lors du gonflement de l'échantillon $A$ et explique également la diminution des contraintes axiale et radiale de gonflement des échantillons $\mathrm{C}$ et $\mathrm{E}$ : il y a réarrangement des agrégats et fermeture plus ou moins marquée des discontinuités.
Dans le cas des argilites litées (B, D) où les paramètres physiques, chimiques, minéralogiques et les courbes de gonflement sont voisins, nous pouvons penser que les comportements observés sont fonction des microtextures initiales et finales. En effet, au départ ces microtextures sont relativement serrées et denses puis, avec le gonflement il y a création d'ouvertures entre les feuillets argileux qui composent les agrégats. Ce phénomène d'expansion est compensé par la fermeture simultanée du réseau de discontinuités initial, c'est-à-dire des espaces inter-agrégats. De ce fait il n'est pas étonnant d'observer une augmentation des contraintes axiales et une stabilisation des contraintes radiales pendant l'essai de gonflement. Les modifications de microtextures sont moins importantes pour les argilites litées que dans le cas des argilites massives. Cela entraîne une moins grande influence sur le mécanisme propre du gonflement et celui-ci est mieux marqué. La microtexture conditionne et oriente donc le phénomène du gonflement (augmentation de la contrainte axiale, diminution ou stabilisation de la contrainte radiale).

Ainsi, l'observation des microtextures au microscope électronique à balayage permet de donner une explication des mécanismes du gonflement de divers matériaux argileux. Le rôle essentiel joué par les microtextures lors du gonflement est mis en évidence et il est ainsi possible d'obtenir une meilleure interprétation entre les différents paramètres mesurés lors de tels essais. Ces conclusions sont en accord avec celles de TOVEY, FRYDMAN et WONG (6).

\section{BIBLIOGRAPHIE}

1. ABOUSHOOK, M. (1978). - Geological and mineralogical factors affecting engineering of expansive soils at Nasr City, Cairo, Egypt. M. Sc. Thesis, Al Azhar University, Le Caire, Egypte.

2. ABOUSHOOK, M. et TISOT J.P. (1983). Application d'une nouvelle méthode pour déterminer le comportement au gonflement des sols intacts. Compte rendu des $2^{\text {es }}$ Journées Universitaires *Génie Civil „, Institut National Polytechnique de Lorraine, E.N.S.G., Nancy, 19-20 octobre 1983.

3. EL SOHBY, M.A.; SALAH, S, et ABOUSHOOK, M. (1981). - X-ray study of Nasr city soils. Journal of the Deserte Institute, Le Caire, Egypte, pp. 1-6.

4. MAZEN, S.O. (1978). - Some geotechnical properties of Nasr City. M. Sc. thesis, Al Azhar University, Le Caire, Egypte.

5. TISOT, J.P. et ABOUSHOOK, M. (1983). Triaxial study of the swelling characteristics. In Proc. of the 7th Asian Regional Conference on soil mechanic and Found. Eng., Haifa, Israèl, vol. 1, pp. 74-78.

6. TOVEY, N.K., FRYDMAN, S. et WONG, T.K. (1973). - A study of a swelling clay in the Scanning Electron Microscope. In Proc. of the 3rd Int. Conference on expansive soils. Haifa, Israël, vol. 2 , pp. $45-55$. 
7. TROALEN, J.P. (1978), - Propriétés physiques et géotechniques des argiles fissurées du Domérien de la région de Nancy. Thèse présentée à l'Institut National Polytechnique de Lorraine, soutenue le 25 juillet 1978 pour obtenir le grade de Docteur $3^{e}$ cycle, pp. 120-122.

8. TROALEN, J.P. (1982). - Les argilites surconsolidées du Domérien de Nancy: relations entre la consolidation, la diagenèse et quelques propriétés géotechniques. Revue Française de Géotechnique, $n^{\circ} 21$, novembre 1982 , pp. 67-73 et p. 73 .

Tableau II:

MITCHELL, J.K. (1973), - Recent advances in the understanding of the influences of mineralogy and pore solution chemistry on the swelling and stability of clays.

Proc. 3rd Int. Conf. on Expansive soils Hailfa, vol. 2 , p. $11-25$

Tableau III :

HOLTZ, W.G. et GIBBS, H.J. (1956). - Engineering properties of expansive clays.

Transactions, A.S.C.E., vol. 121, p. 641-677.

RANGANATHAM, B.V et SATYANARAYANA, B. (1965). - A Rational method of predicting swelling potential for compacted Expansive clays.

Proc. 6th I.C.M.S., Montréal, p. 92-96.

SKEMPTON, A.W. (1953): - The colloidal Activity of clays.

Proc. 3rd I.C.M.S., Suisse, vol. 1, p. 57-61. 